\title{
Independent Component Analysis of Spectral Absorbance Image in Human Skin
}

\author{
Norimichi Tsumura, Hideaki Haneishi and Yoichi Miyake \\ Department of Information and Image Sciences, Chiba University, \\ 1-33, Yayoi-cho, Inage-ku, Chiba 263-8522, Japan
}

(Revised June 20, 2000; Accepted September 12, 2000)

The image of spectral absorbance in human skin is analyzed by independent component analysis. The spectral absorbance image is estimated from an image with red, green, and blue channels. The qualitative information and spatial distribution of the pigments can be extracted by the independent component analysis. It is shown that the extracted absorption characteristics of the pigments are corresponding to those of hemoglobin and melanin.

Key words: skin pigmentation, independent component analysis, in vivo, melanin, hemoglobin, spectral absorbance image, spectral analysis

E-mail: tsumura@ics.tj.chiba-u.ac.jp 


\section{Introduction}

Extracting qualitative information and spatial distribution of components through analysis of an observed image is required in many fields of image analysis such as remote sensing, medical diagnostics, and robot vision. Spectral characteristics of the components are useful information to identify those components, because different materials have different spectroscopic responses to electromagnetic waves of a certain energy band. A technique that uses a spectral image as an observed image was proposed 1 and is expected to lead the field of image analysis.

Skin color reproduction is considered the most important problem in color reproduction of color film and color television systems. With the recent progress of various imaging systems ${ }^{2-4}$ such as multimedia, computer graphic and telemedicine systems, skin color becomes increasingly important for communication, image reproduction on hardcopy and softcopy, medical diagnosis, cosmetic development and so on. Human skin is a turbid medium with multi-layered structure 5,6 , and contains various pigments such as melanin and hemoglobin. Slight changes in structure and pigment construction produce great skin color variation. This make it necessary to analyze skin color on the basis of its structure and pigment construction in reproducing and diagnosing various colors.

In this paper, an image of spectral absorbance in human skin is analyzed by independent component analysis (ICA) to extract information of the components in the skin. ICA is a technique that extracts the original signals from mixtures of many independent sources without a priori information on the sources or the process of the mixture. ICA has been applied to problems of array processing, communication, medical signal processing, and speech analysis 7 . In the field of color image processing, Inoue et al. 8 proposed a technique to separate each pigment from compound color images. In a previous paper ${ }^{9}$, we improved on their technique and applied it to skin color 
image with red, green, and blue color channels, and separated the image into the spatial distributions of hemoglobin and melanin. The pixel values of three color channels are dependent on the imaging device, so that it was impossible to analyze the separated colors directly. The spectral absorbances which are not affected by the illuminant and imaging system are analyzed in this paper.

In Section 2, the skin spectral reflectance image is obtained from skin color image by the technique described in references 2 and 10 , and transformed to a spectral absorbance image. In Section 3, the application of ICA to the color image separation is reviewed based on references 8 and 9. In Section 4, skin spectral absorbance is modeled on the basis of the two pigments, melanin and hemoglobin. The results of independent component analysis for skin spectral reflectance images are shown in Section 5.

\section{Spectral Absorbance Image in Human Skin}

The skin color image with 64 x 64 pixels is used in this paper, as was used in our previous paper ${ }^{9}$. The image is extracted from the forehead of a Mongoloid facial image with $300 \times 450$ pixels taken by HDTV camera(Nikon HQ1500C) with $1920 \times 1035$ pixels $^{9}$ under a metal halide lamp with color temperature of $5,700 \mathrm{~K}$. The extracted region of the forehead is almost uniformly illuminated. Each pixel of these color images has three channels: red, green and blue. Nonlinearity between RGB levels and luminance was compensated to be linear ${ }^{2}$.

The spectral reflectance is estimated from the $R, G$, and $B$ values in each pixel of skin image using the low-dimensional linear approximation method for spectral reflection 2,10 to obtain the spectral reflectance image. Smoothness of the spectral reflectance curves is effectively used in the low-dimensional linear approximation, and the spectral characteristics of the illuminant and imaging system are calibrated using thirty-nine patches of Japanese skin color ${ }^{2}$. The estimated reflectance spectra are transformed to spectral absorbances (optical density) by logarithm transformation. Figure 1 shows forty examples of the 
estimated spectral absorbances in the skin image. Each absorbance corresponds to a pixel in the image.

\section{Independent Component Analysis for Color Image Separation}

The application of independent component analysis to the color image separation 8,9 is briefly reviewed in this section. For a simple explanation, it is assumed that the medium is constructed of two pigments and captured by an imaging system with two color channels, or captured at any two wavelengths.

Let $\boldsymbol{e}_{l, m}$ and $\boldsymbol{x}_{l, m}$ denote the two-dimensional compound color vector and quantity vector on the image coordinate $(l, m)$, respectively. It is assumed the two vectors have the following relationship with the transformation matrix A :

$$
\begin{aligned}
& \boldsymbol{e}_{l, m}=\mathrm{A} \boldsymbol{x}_{l, m}, \\
& \mathrm{~A}=[\boldsymbol{a}(1), \boldsymbol{a}(2)]
\end{aligned}
$$

where A denotes the mixing matrix, and $\boldsymbol{a}_{(1)}$ and $\boldsymbol{a}(2)$ denote pure color vectors of the two pigments per unit quantity. By applying the ICA to the compound color vectors in the image, the relative quantity and pure color vector of each pigment are extracted without a priori information on these items, by assuming that quantities of pigments are mutually independent in the image coordinate. In performing ICA, let us define the following equation using the separating matrix H and separated vector $\boldsymbol{S}_{l, m}$.

$$
\boldsymbol{S}_{l, m}=\mathrm{H} \boldsymbol{e}_{l, m},
$$

By finding the appropriate separating matrix $\mathrm{H}$, we can extract the mutually independent signals as elements of vector $\boldsymbol{S}_{l, m}$ from the compound color vectors in the image. Many methods for finding a separating matrix have 
been proposed(for example, Refs. 11-13). It is noted that the extracted signals and inverse matrix of $\mathrm{H}$ correspond to the elements of quantity vectors $\boldsymbol{x}_{l, m}$ and mixing matrix $\mathrm{A}$, respectively.

\section{Skin Spectral Absorbance Model}

In analyzing skin spectral absorbances, we made three assumptions. First, we assumed that the Lambert-Beer law or the modified Lambert-Beer law 14 holds in skin absorbances. Second, the spatial variations of color in the skin are caused by two pigments: melanin and hemoglobin. Third, these quantities are spatially independent of each other.

On the basis of the first and second assumptions, the spectral absorbance in the skin image is modeled on the basis of linear combination of the absorption coefficients of melanin and hemoglobin as shown in Fig. 2 , where only three absorbances $\mu_{a}\left(\lambda_{1}\right), \mu_{a}\left(\lambda_{2}\right), \mu_{a}\left(\lambda_{3}\right)$ at three wavelengths $\lambda_{1}, \lambda_{2}, \lambda_{3}$ are shown for convenience. As shown, the absorbances of skin are distributed on a two-dimensional plane spanned by pure spectral vectors of melanin and hemoglobin. This two-dimensional plane can be extracted by principal component analysis, and the resultant two dimensional plane is then used for the rest of the analysis ${ }^{15}$. Therefore, Eq. (1) can be directly applied to the two dimensional plane for independent component analysis.

\section{Experimental Results}

The total flowchart of the image analysis is shown in Fig. 3. In the principal component analysis of spectral absorbance, the resultant cumulative contribution ratio for the first and second principal components was 0.90. This matches the model of spectral absorbance shown in Fig.2. Each element of the separating matrix $\mathrm{H}$ in Eq.(3) was obtained by minimizing the Burel's independence evaluation value 11 for the elements of vector $\boldsymbol{S}_{l, m}$. This ranges 
from 0 to 1 , and if the value is 0 , the signals are mutually independent. The minimization was performed by quasi-Newton implementation using the MATLAB tool box ${ }^{16}$. The independence value for the resultant signals was 0.0073. Highly independent signals were extracted as the resultant signals. In a previous paper where the image with $\mathrm{R}, \mathrm{G}$, and B channels was used, we found that the independence value for the resultant signals was 0.0081 . We can conclude that the resultant independence is improved by the linearity of the spectral absorbance model because the spectral density domain was used in the analysis rather than the optical density domain of three channels. Figure 4 shows original skin color image with $64 \times 64$ pixels and images of color densities for the first and second independent components. It is assumed that the two independent components are caused by hemoglobin and melanin, respectively, since the pimples are seen in the first independent component and are not seen in the second.

Figure 5 shows the extracted pure spectral absorbances, which are $\boldsymbol{a}_{(1)}$ and $\boldsymbol{a}(2)$ in Eq. (1) in the spectral density domain. Figure 6 shows the spectral absorption characteristics of hemoglobin and melanin which were measured in vitro by Anderson and Parrish 6 . The spectral absorbance of the first independent component in Fig. 4 is similar to that of hemoglobin in Fig. 6, especially at the two typical absorptions around $540 \mathrm{~nm}$ and $580 \mathrm{~nm}$, and that of the second component is similar to the absorbance of melanin. We can conclude that ICA effectively extracts the two pigment components of skin: hemoglobin and melanin. It is noted that unknown pure spectral absorbances are extracted from a skin image which was taken in vivo.

\section{Conclusion and Discussion}

We have extracted the spectral characteristics of the components, hemoglobin and melanin in human skin by independent component analysis of a spectral absorbance image without a priori information. These extracted absorbances 
are influenced by the distribution of optical path length in skin caused by optical scattering. The scattering coefficient of skin is higher in the short wavelength ${ }^{5}$, that is, the mean optical path length is shorter in the short wavelength. This will effect the result of the extracted pure spectral absorbances. We consider that this scattering effect and melanin content are the reason strong absorbance around $430 \mathrm{~nm}$ in Fig. 6 is not seen in Fig. 5. From a different point of view, the extracted pure spectral absorbances will provide information about the wavelength dependent characteristic of optical scattering when pure absorbances in vivo are known.

The broad distribution of the optical path length histogram in the skin may also violate the linearity in Eq. (1). In near infrared spectroscopy, it is found that the linearity holds in measuring human tissue. This characteristic is called either the modified or the macroscopic Lambert Beer law ${ }^{17}$. However, in the visible range of wavelength, it is not certain whether this linearity holds in measurement of the human tissue. In the visible range, strong absorption may violate the linearity, and we believe that the abnormal low absorption around $570 \mathrm{~nm}$ in the extracted melanin component is an artifact from the corresponding hemoglobin absorption band present in the first component. Hemoglobin has two states: oxy-hemoglobin $\left(\mathrm{HbO}_{2}\right)$ and deoxy-hemoglobin $(\mathrm{Hb})$. The spectral absorptions are different from each other, and the ratio between $\mathrm{HbO}_{2}$ and $\mathrm{Hb}$ will change spatially ${ }^{18}$. This may also cause abnormal absorption.

\section{References}

1) S. Kawata, K. Sasaki and S. Minami: J. Opt. Soc. Am. A 4 (1987)2101.

2) F. H. Imai, N. Tsumura, H. Haneishi and Y. Miyake: J. Image Sci. Technol. 40(1996)422.

3) Y. Yokoyama, N. Tsumura, H. Haneishi, Y. Miyake, J. Hayashi and M. Saito: Proceedings of IS\&T/SID's 5th Color Imaging Conference, Color Science, 
Systems and Appl. (Society for Imaging Science \& Technology, Springfield, VA, 1997) pp. 169-172 .

4) M. Yamaguchi, R. Iwama, Y. Ohya, T. Obi, N. Ohyama and Y. Komiya: Proc. Soc. Photo-Opt. Instrum. Eng. 3031(1997)482.

5) M.J.C.van Gemert, S.L. Jacques, H.J.C.M. Sternborg and W.M. Star: IEEE Trans. Biomed. Eng. 36(1989)1146-.

6) R.R. Anderson and J.A. Parrish: J. Invest. Dermatol. 77 (1981)13.

7) J. Karhunen, A. Hyvarinen, R. Vigário, J. Hurri and E. Oja: Proceedings of IEEE International Conference on Acoustics, Speech, and Signal Processing(IEEE Computer Society Press, Los Alamitos, Calif. 1997), Vol. 1, 131-134.

8) T. Inoue, Y. Fujii, K. Itoh and Y. Ichioka: 1996 International Topical Meeting on Optical Computing. Technical Digest(The Japan Society of Applied Physics, Tokyo, 1996) Vol.1, p.40.

9) N. Tsumura, H. Haneishi and Y. Miyake,: J. Opt. Soc. Am. A 16(1999)2169.

10) M.J. Vrhel and H.J. Trussell: Color Res. Appl. 17(1992)328.

11) G. Burel: Neural Networks 5 (1992)937.

12) J. Karhunen, E. Oja, L. Wang, R. Vigário and J. Joutensalo: IEEE Trans. Neural Network 8(1997)486-.

13) C. Jutten and J. Hearult: Signal Process. 24(1991)1.

14) M. Hiraoka, M. Firbank, M. Essenpreis, M. Cope, S.R. Arridge, P. van der Zee and D.T. Delpy: Phys. Med. Biol. 38 (1993)1859.

15) K. Shirasaki, T. Inoue, K. Itoh and Y. Ichioka: Jpn. J. Opt. (KOGAKU) 25 (1996)537(in Japanese).

16) A. Garce. Matlab Optimization Toolbox User's Guide (The Math Works, Boston, MA, 1992).

17) Y. Nomura, O. Hazeki and M. Tamura: Phys. Med. Biol. 42(1997)1009.

18) P.J. Dwyer, R.R. Anderson and C.A. DiMarzio: Proc. Soc. Photo-Opt. Instrum. Eng. 2976(1997)270. 


\section{Figure Captions}

Fig. 1. Forty examples of estimated spectral absorbances.

Fig. 2. Model of spectral absorbance in human skin.

Fig. 3. Flowchart for the analysis of the human skin image.

Fig. 4. Separation of skin image (a)skin color image with 64 x 64 pixels, images of color densities for the (b) first and (c) second independent components.

Fig. 5. Extracted pure spectral absorbances.

Fig. 6. Absorption characteristics of hemoglobin and melanin measured in vitro 6. 